\title{
Modelo VHDL de Control Neuronal sobre tecnología FPGA orientado a Aplicaciones Sostenibles
}

\author{
VHDL Neural Control Model on FPGA technology oriented \\ to Sustainable Applications \\ Cecilia Sandoval-Ruiz ${ }^{1 *}$
}

Recibido 19 de Octubre de 2017, aceptado 20 de agosto de 2018

Received: October 19, 2017 Accepted: August 20, 2018

\begin{abstract}
RESUMEN
El presente trabajo consta de una investigación en esquemas de control neuronal y el diseño generalizado de sus componentes en lenguaje descriptor de hardware VHDL, con el propósito de construir un modelo para el soporte de control reconfigurable y la optimización de estos esquemas para implementación con tecnología FPGA. El método seleccionado consistió en el modelado del control, a través de la configuración VHDL de las redes neuronales aplicadas, el estudio de los modelos y la propuesta de entrenamientos más eficientes, orientados a hardware. Entre los resultados se cuenta con una propuesta de configuración fractal para el control eficiente de los sistemas y su modelo matemático. La investigación plantea el concepto de redes neuronales fractales, control neuronal reconfigurable y sistemas de entrenamiento para hardware como un aporte para abordar el control de los sistemas de potencia y reconfiguración de la infraestructura energética. El esquema de control propuesto simplifica la implementación de control avanzado, promoviendo un área de investigación en esta línea de diseño sostenible, incorporación de energías renovables, reutilización de recursos y eficiencia energética.
\end{abstract}

Palabras clave: Tecnología de control, modelos de redes neuronales, identificación de sistemas, algoritmos adaptativos, control óptimo, redes eléctricas inteligentes.

\begin{abstract}
The present work consists of a research on neuronal control schemes and the generalized design of its components in VHDL hardware descriptor language, with the purpose of building a model for reconfigurable control support and the optimization of these schemes for implementation with FPGA technology. The selected method consisted in the modeling of the control, through the VHDL configuration of the neural networks applied, the study of the models and the proposal of more efficient trainings, oriented to hardware. Among the results we have a fractal configuration proposal for the efficient control of the systems and their mathematical model. The research proposes the concept of fractal neural networks, reconfigurable neuronal control and training systems for hardware as a contribution to address the control of power systems and reconfiguration of the energy infrastructure. The proposed control scheme simplifies the implementation of advanced control, promoting an area of research in this line of sustainable design, incorporation of renewable energies, reuse of resources and energy efficiency.
\end{abstract}

Keywords: Control technology, neural network model, system identification, adaptive algorithms, optimal control, smart grid.

\footnotetext{
1 Facultad de Ingeniería. Instituto de Matemática y Cálculo Aplicado IMYCA. Universidad de Carabobo. Venezuela. E-mail: cesandova@gmail.com

* Autor de correspondencia: cesandova@gmail.com
} 


\section{INTRODUCCIÓN}

Actualmente, los sistemas de conversión de energías renovables enfocados a redes eléctricas inteligentes, requieren de esquemas de control adaptativos, que demandan capacidad de cómputo y algoritmos particulares, sobre los que se centran recientes investigaciones [1].

Los sistemas eléctricos de potencia y los elementos de conversión de energía presentan una dinámica compleja, cuya optimización viene dada a partir del esquema de control. Un aspecto primordial en el diseño es la posibilidad de adaptación del esquema y los parámetros del controlador, a fin de garantizar su eficiencia. Se plantea un modelo neuronal configurable para soporte de esquemas de control, orientados al menor consumo energético, por lo que se pueden considerar principios de control óptimo [2] y control predictivo [3].

Por otra parte, la plataforma de implementación puede ser determinante, siendo la tecnología FPGA - Field Programmable Gates Array, considerada por su flexibilidad y alta capacidad de cómputo. Un modelo general bajo tecnología de hardware reconfigurable, se perfila como una solución. Esto, ya que se requiere controlar diversos elementos, cuya combinación permita obtener la salida deseada, a partir del aporte ponderado de las entradas, que debe ajustarse a las condiciones ambientales.

\section{Antecedentes}

La investigación presentada en [4], define una arquitectura para la configuración de esquemas circuitales, que permiten la RDP - reconfiguración dinámica parcial, con mayor flexibilidad en la configuración topológica de las redes neuronales. Esto con el fin de favorecer la reutilización de procesadores neuronales, usados en una misma área de circuito, siendo los módulos de procesamiento neuronal idóneos en el diseño de esquemas de control avanzado, por sus características de procesamiento paralelo.

El tema de control híbrido conmutado sobre tecnología FPGA para eficiencia energética en sistemas convertidores de potencia es abordado por [5], en el que se estudian los casos de control deslizante y control óptimo híbrido, como algoritmos de control para un modelo de decisión implementado sobre un dispositivo de hardware reconfigurable.
Describiendo una metodología en la que se define la ley de control y se realiza un análisis numérico, a través de simulaciones previas en software.

Diversos trabajos presentan el diseño de esquemas de control óptimo [2], control adaptativo [6], redes neuronales en estimación de la demanda, control de componentes de conversión de energía [7] y algoritmos de entrenamiento de redes neuronales en hardware, para control de aplicación sostenibles [8]. Todo esto demuestra la necesidad de un algoritmo de control generalizado, orientado a aplicaciones de tecnologías sostenibles, como elementos de conversión de energía renovable en centrales eléctricas y redes distribuidas - Smart Grid.

\section{Campo de Aplicación}

En el estudio de las aplicaciones se consideran los diseños de sistemas sostenibles. A partir de una plataforma de convertidores de energía renovable, aplicando hardware reconfigurable en la etapa de control [9-10], el diseño de algoritmos adaptativos en VHDL - Hardware Description Language [6], [8], neurocontrol para sistemas de conversión de energía eólica SCEE [11]. Lo que permite observar la alternativa de control neuronal y reconfigurable como solución a sistemas dinámicos de energía renovable y otros sistemas de dinámica compleja.

De esta manera, se propone la incorporación del control neuronal reconfigurable en sistemas de redes eléctricas adaptativas [12-16], dispositivos electrónicos inteligentes [17], reconfiguración dinámica de la arquitectura de los elementos del sistema de generación, materiales fotovoltaicos, materiales termoeléctricos y arreglos de convertidores inteligentes.

Es así como se define el área de investigación que propone alternativas de solución en materia energética, bajo el concepto de redes reconfigurables, para establecer la configuración más eficiente de la red eléctrica, incorporando control e inteligencia artificial [15-16]. La aplicación de este modelo de control electrónico para gestión de la infraestructura energética inteligente, comprende medición, control avanzado, energías renovables y comunicación de soporte para configuración remota.

En esta investigación se propone un modelo y su algoritmo de soporte para la reconfiguración de la 
arquitectura y control, basado en el principio de esquemas de control híbrido. Al mismo tiempo que se busca optimizar el consumo de componentes hardware, en el dispositivo de implementación. El modelo de control reconfigurable deberá soportar esquemas de control avanzado, integrando control óptimo, adaptativo y predictivo, bajo un modelo generalizador como son las redes neuronales. Este arreglo puede ser planteado sobre un sistema de funciones iteradas del modelo matemático, que se describe en lenguaje descriptor de hardware, para su implementación en circuito.

\section{Motivación de la Investigación}

El objetivo de este trabajo de investigación es presentar un modelo que permita simplificar la configuración, entrenamiento e implementación de las redes neuronales sobre FPGA, aplicadas a control avanzado de sistemas de energías renovables. Con el propósito de ampliar el alcance de los sistemas sostenibles y promover estas aplicaciones, disminuyendo costos de operación, reutilización de HW y eficiencia, bajo criterios de responsabilidad ambiental.

La investigación plantea el concepto de redes neuronales fractales, como un aporte para abordar el control de los sistemas, en componentes neuronales de estructura auto-similar, que puedan ser entrenadas de forma modular. Además del entrenamiento fraccionado como un novedoso método práctico, con la ventaja de un esquema modular, que permite aprovechar la capacidad de reconfiguración parcial dinámica de la tecnología FPGA.

\section{ANÁLISIS TEÓRICO}

Un sistema de control neuronal embebido en un FPGA, requiere la configuración de los módulos de control neuronal en lenguaje descriptor de hardware, estableciendo la relación entre las señales de control del sistema y su interacción con las capas de la red neuronal. En la que se debe definir la arquitectura, número de capas, neuronas y sus características, seguidas del algoritmo de adaptación, presentado en Figura 1.

Se puede observar los componentes neuronales descritos en VHDL, así como el algoritmo de entrenamiento del controlador neuronal, con lo que se obtienen los parámetros de la red neuronal que minimiza el error en la salida deseada (target) de la planta. En tal sentido, partiendo del comportamiento deseado del sistema a controlar se realiza el diseño $\mathrm{y}$ entrenamiento del control neuronal para hardware reconfigurable.

En los modelos de los sistemas de control, se encuentra una etapa de modelado, asociado a estrategias de

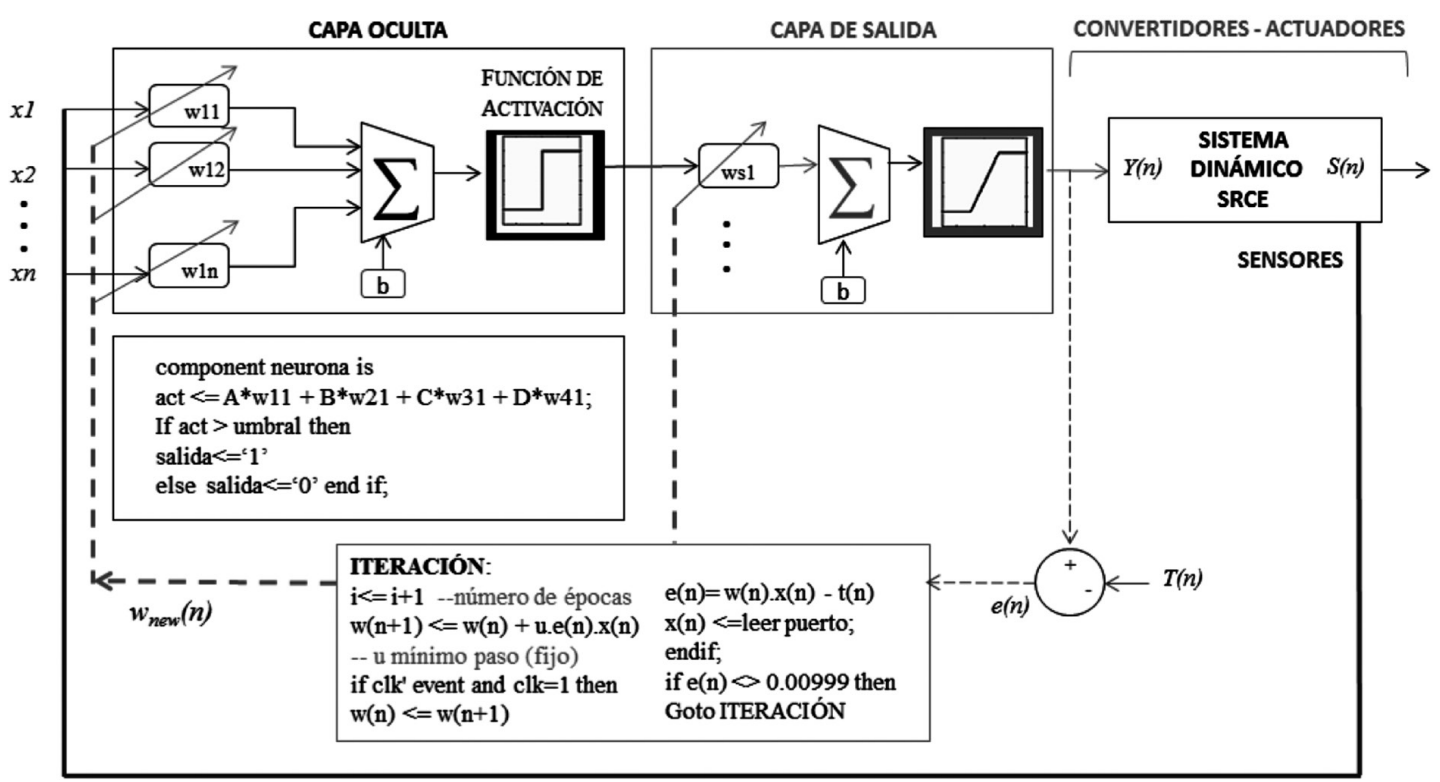

Figura 1. Modelo de Red Neuronal Reconfigurable. 
control predictivo [18], en este caso un modelo neuronal de la planta y un módulo de optimización el cual determina el valor de la entrada $u(t)$ que minimiza la energía $J$, obteniendo el valor óptimo de la entrada a la planta. Este proceso consta de una red neuronal, entrenada por lotes, para establecer el modelo de planta, prediciendo los valores futuros de la salida del sistema y un algoritmo de optimización, entrenado de forma incremental, para hallar los parámetros de módulo optimizador, lo que requiere más capacidad de cómputo para su implementación en hardware.

Considerando estas características y las limitaciones correspondientes a la demanda de cómputo por parte del control neuronal con algoritmo de optimización, se plantea una alternativa dada por el entrenamiento con algoritmo de optimización sobre una red neuronal dinámica, la cual puede representar el controlador de manera eficiente sobre el hardware del FPGA.

\section{Modelo Predictivo}

Este modelo [19] aplica una red neuronal para predecir el comportamiento de una planta no lineal (sistema a controlar), a fin de estimar las salidas futuras del sistema. De esta manera, en la primera etapa se tiene un identificador de sistema que modela la planta de acuerdo a las entradas-salidas de ésta y en la segunda etapa el optimizador que estima la señal de entrada a la planta para cumplir con la salida óptima, como se observa en la Figura 2.

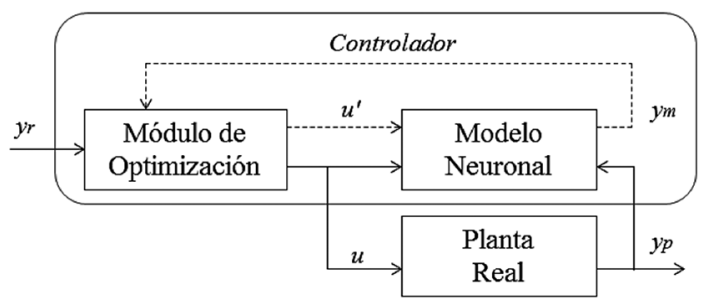

Figura 2. Control Predictivo con Modelo Neuronal [19].

En este control se presentan las señales de entrada $y_{r}$ : respuesta deseada, $y_{p}$ : salida de la planta, y la entrada $u$ : entrada de la planta (salida del controlador). En tanto que las señales internas del controlador corresponden a $u$ ': señal de entrada al modelo neuronal de la planta y, $y_{m}$ : salida del modelo neuronal de la planta (predictor), a fin de corregir en el módulo de optimización el comportamiento de la planta real.
Los parámetros de la red a ajustar corresponden al módulo de optimización y la red del modelo de la planta, variando los retardos asociados a las entradas y el número de neuronas de la capa oculta.

\section{Modelo Neuronal de Identificación de Sistemas}

En este módulo se definen las características de la red: número de neuronas de la capa oculta, los delays para cada una de las entradas de la red (entrada y salida de la planta), y las características de la data de entrenamiento: número de muestras y el modelo de la planta con la cual se obtienen los mencionados datos.

\section{Control de Modelo de Referencia}

Este esquema presenta una etapa de reconocimiento de la planta y un controlador. Las señales de entrada del controlador corresponden a $y_{r}(t)$, respuesta deseada (salida del modelo de referencia), esta señal será comparada con la salida de la planta $y_{p}(t)$, obteniendo el error de la planta controlada $e_{c}(t)$. La red del controlador no recibe en este esquema la salida del modelo neuronal, como en el caso del predictor $y_{m}$, en tanto que esta salida es utilizada para calcular el error del modelo neuronal $e_{p}(t)$.

\section{Modelo Neuronal Reconfigurable}

Para establecer el modelo del sistema de forma selectiva, se plantea la red neuronal como un estimador del comportamiento del sistema (elementos de conversión de energía renovable a modelar), la diferencia entre la salida de la red neuronal $y_{m} \mathrm{y}$ la salida real de la planta $y_{p}$, se realimenta como el error para el algoritmo de entrenamiento de la red. La red neuronal para el modelado del sistema tiene como entradas: $y_{r}(n)$, correspondiente a la respuesta deseada (según los criterios de control seleccionados) y, $y_{p}(n)$, respuesta de la planta, esquema presentado en la Figura 3.

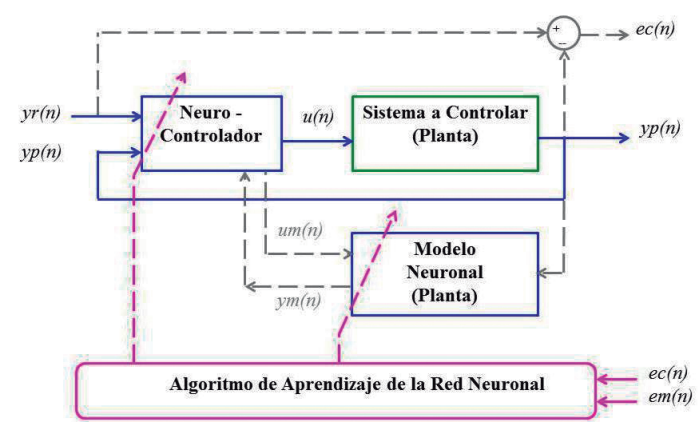

Figura 3. Esquema de Control Neuronal. 
En este esquema se observa la topología general del controlador neuronal, el cual comprende una RNA, como modelo de la planta, cuyas entradas vienen dadas por la salida del controlador según la configuración de control (donde las salidas del neurocontrolador, serán las señales de accionamiento de la planta $u(n), u_{m}(n)$, esta última señal corresponde a la entrada para optimizar la función o minimizar la energía $J$ ), igualmente la salida del modelo neuronal de la planta $y_{m}(n)$, se realimenta al módulo de control solo en el caso de control predictivo, para el cual esta señal corresponde a la estimación de la salida futura de la planta $y_{p}(n+1)$.

El error de la salida de la planta $y_{p}(n)$, se calcula con respecto a la respuesta deseada $y_{r}(n)$, obteniendo así el error $e_{c}(n)$, que será el criterio del algoritmo de aprendizaje de la red para establecer los parámetros del controlador. Por su parte el error del modelo neuronal $e_{m}(n)$, se estima de la comparación entre la salida de la planta $y_{p}(n)$ y la salida del modelo neuronal $y_{m}(n)$, siendo éste el criterio del algoritmo de aprendizaje para la red neuronal del modelo del sistema. De esta manera, se tiene el modelo de la planta que será estimado y con esa etapa de reconocimiento del sistema se obtendrán los parámetros de la red neuronal.

\section{Redes Neuronales Fractales}

Una red neuronal con todas las entradas conectadas directamente a las neuronas de la capa de salida se denomina red neuronal de una sola capa, ya que cada unidad de salida es independiente de las otras, si la red neuronal está ordenada en etapas se presenta una arquitectura multicapas MPL, el número de capas define el nivel de profundidad de la red, que puede ser simplificada con una red fractal.

El esquema de una red neuronal lineal, con topología circuital fractal [9], en la que la operación de un componente está definida por una subred de parámetros ajustables, con algoritmo adaptativo para el cálculo de los pesos sinápticos de la subred neuronal responsable de definir la operación de convolución a través de registros desplazamientos [21] de los operadores complejos de la red neuronal reconfigurable [22], que corresponden a los componentes principales en el esquema fractal, presentado en la Figura 4.

En el caso de múltiples neuronas (en una sola capa), se tendrá una matriz de pesos relacionados

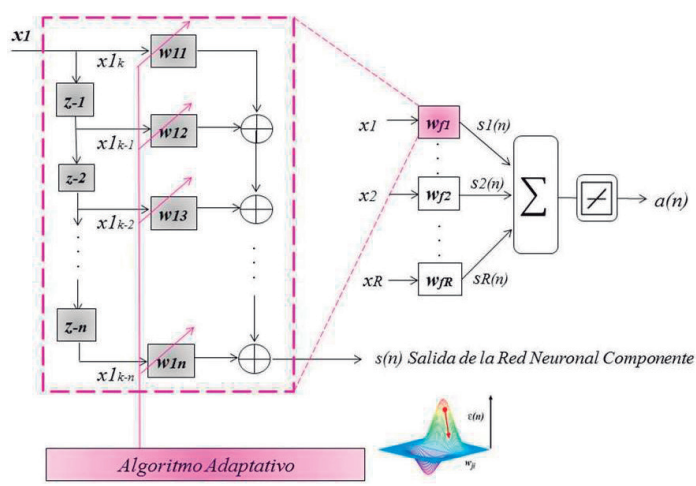

Figura 4. Modelo de Control con Red Fractal.

a cada conexión entre las entradas y las neuronas correspondientes, como se presenta en la ecuación (1).

$$
f\left(\sum_{i=1}^{k} w_{j, i} \cdot p_{i}+b\right)
$$

Siendo: $w_{j, i}$ el peso de la conexión entre la entrada $i$ y la neurona $j, p_{i}$ la entrada $i$ a la neurona, $b$ el sesgo. La neurona se comportará como la unidad básica de procesamiento de la red neuronal, siendo definido su modelo matemático generalizado, por la sumatoria ponderada de las entradas, cuya implementación comprende operaciones de producto de las entradas por lo pesos de las conexiones. Para una neurona, como se describe en la ecuación (2).

$$
a(n)=f\left(w_{1} \cdot p_{1}+w_{2} \cdot p_{2}+\ldots+w_{k} \cdot p_{k}\right)
$$

La red adaptativa lineal puede ser configurada para diversas aplicaciones, como un aproximador de funciones, filtro adaptativo lineal, predictor lineal adaptativo, para los cuales se incorpora una línea de retardo en la entrada. En tanto que la red con las entradas concurrentes (red estática) se tiene la salida dada por la ecuación (3).

$$
a=\left(\sum_{i=0}^{R} w_{j, i} p_{i}\right)
$$

Con la secuencia de entrada $p(k)$ como entrada a la red dinámica (con línea de retardo) se tiene la salida dada por la ecuación (4).

$$
a(k)=\sum_{i=0}^{R} w_{j, i} p_{i}(k-i+1)
$$

Con $p(k)$ como Target se tiene la salida dada por la ecuación (5). 


$$
a(k)=\sum_{i=0}^{R} w_{j, i} p_{i}(k-i)
$$

El entrenamiento se realiza a través del algoritmo de aprendizaje, notando que el algoritmo permite adaptar los pesos sinápticos para obtener una salida deseada, a través de la ponderación de las entradas, este tipo de control adaptativo presenta similitud entre algoritmos LMS para beamforming [23] y para arreglos de paneles solares, se calcula el valor de los pesos según la ecuación (6).

$$
w_{\text {new }}=w_{\text {old }}+\alpha\left(t_{q}-\alpha_{q}\right) p_{q}^{T}
$$

En el caso de las Deep Neural Network - DNN, un algoritmo de pre-entrenamiento por capas presenta mejores resultados [24]. Entre sus ventajas se encuentra el entrenamiento fraccionado (preentrenamiento supervisado), un novedoso método práctico, que permite aprovechar la capacidad de reconfiguración parcial dinámica de la tecnología FPGA. Siendo un aporte de esta investigación, para las redes neuronales fractales, las cuales pueden ser incluidas como una subclase de las redes neuronales de arquitectura profunda. A través de módulos entrenados para codificar la entrada $x$ en alguna representación correspondiente $c(x), d$ onde se puede establecer la correspondencia con el concepto de auto-encoders.

\section{Sistema Reconfigurable de Conversión de Energías renovables - SRCEr}

Este sistema modular, puede ser configurado, en tiempo de operación, de manera remota [25]. Siendo diseñados como micro-módulos se simplifica su implementación y disminuyen los costos asociados a operación y mantenimiento. Los mismos pueden ser escalados e integrados en micro-redes, diseñando la arquitectura de la red de energía, a partir de las condiciones del entorno, las características de las fuentes de energía renovable y la demanda eléctrica en el tiempo, a fin de optimizar la respuesta del sistema y su eficiencia energética. El sistema dinámico propuesto comprende Sistemas Híbridos de Generación de Energía Eléctrica SHGEE [26], a través de la conversión de energía eólica SCEE [11], fotovoltaica, Termo-Solar, micro-convertidores, biomasa, etc. En los que se incorpora la capacidad de reconfiguración, el modelo neuronal de control avanzado y tecnología FPGA, que se ajusta a la dinámica propia de estos sistemas a través de relés, que permitan acoplar de forma selectiva etapas del sistema eléctrico de potencia, el modelo propuesto es presentado en la Figura 5.

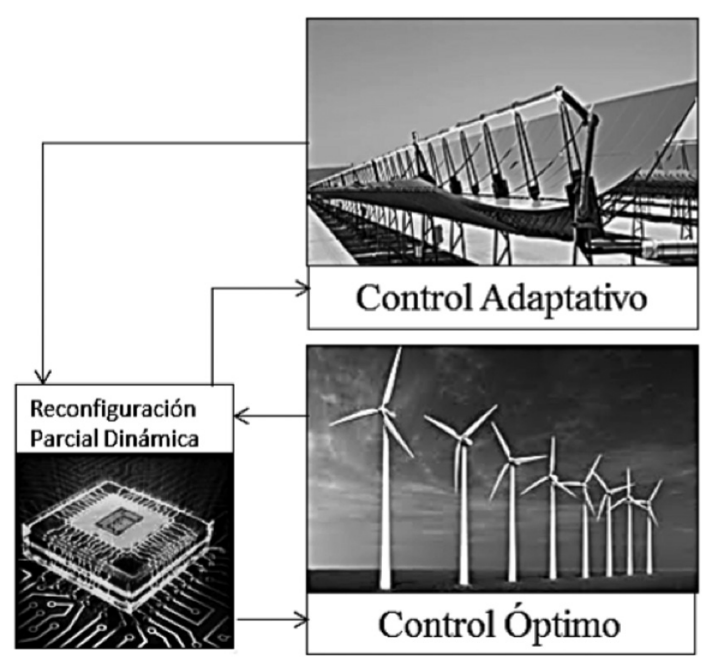

Figura 5. Esquema de Control Reconfigurable.

Los dispositivos electrónicos inteligentes IEDs [17] sobre hardware reconfigurable, permiten el diseño de una arquitectura de control distribuida de la red eléctrica, el diagnóstico de condiciones de la red, monitoreo de requerimiento de la Smart Grid [27], control inteligente y comunicación de señales de control [28-29], a fin de configurar los parámetros en los elementos convertidores, transformadores e interruptores, de forma remota. Entre sus funciones de diseño se encuentran infraestructura de medición avanzada - AMI, gestión de la demanda eléctrica - DMS, auto-reconfiguración de los circuitos de potencia. Siendo parte de un sistema compuesto de automatización, instrumentación y control (red de sensores inteligentes), comunicación (enlaces bidireccionales, gestión remota) y potencia (asignación de fuentes de energías renovables según la demanda).

\section{MÉTODO DE DISEÑO}

La metodología de diseño comprende estudio de esquemas de control, como insumo para el entrenamiento de la red neuronal fractal, modelado, algoritmo de implementación en hardware y finalmente la descripción del esquema de control reconfigurable, la estimación de recursos sobre el FPGA, para su análisis. 
Al momento de definir el sistema a controlar, se analizan aplicaciones en el campo de redes eléctricas con fuentes de energías renovables, como objetivo de la propuesta. Entre estas aplicaciones destaca el control de posición de arreglos colectores solares, utilizados en el proceso de conversión de energía termo-solar, siendo la variable temperatura la seleccionada para medición por su accesibilidad en estos modelos.

\section{Control Adaptativo de Temperatura aplicado en Sistema Termo-Solar}

En la primera descripción se diseñó un control de ganancia adaptativa, presentada en la Tabla 1, en la cual se establece una señal de referencia que corresponde al valor de temperatura deseado (bajo criterios de máxima eficiencia) $x(n)$, la señal de salida será $y(n)$, el coeficiente sobre la variable de control será $c(n)$ que será adaptativo en función del factor $g(n)$.

Tabla 1. Algoritmo de Ganancia Adaptativa.

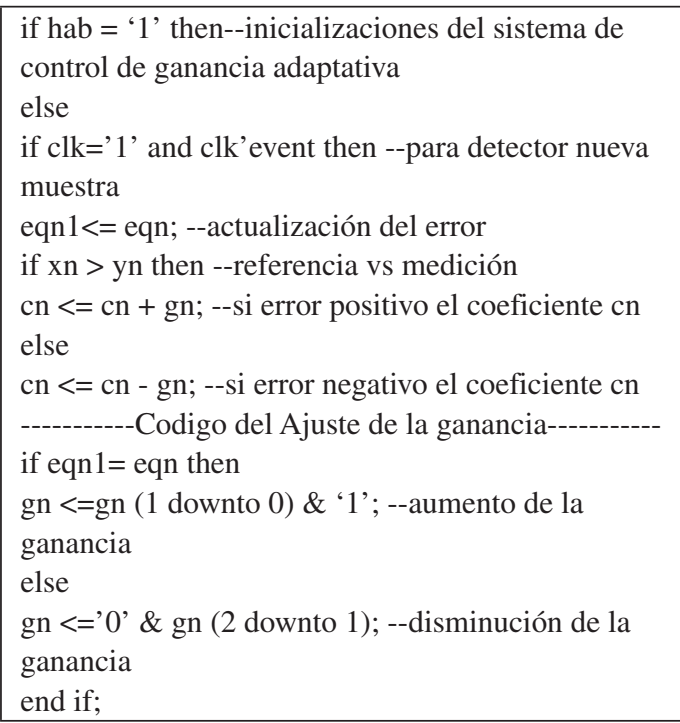

Para el algoritmo de ganancia adaptativa se realizó la simulación, en la cual se obtuvo como respuesta a la entrada de referencia $x(n)$, la salida $y(n)$, mostrada en la Figura 6.

Se pudo observar el seguimiento de la señal, con Delta como la representación del signo del error y $G(n)$ el parámetro adaptativo, se seleccionaron incrementos y decrementos de diversos pasos,

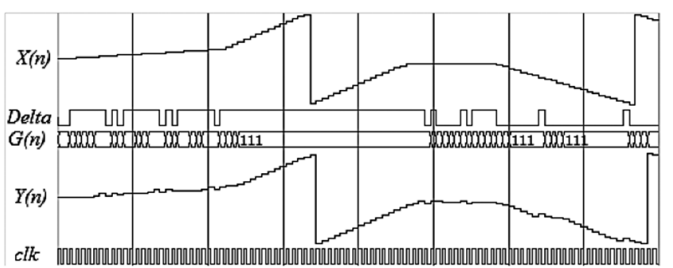

Figura 6. Control Adaptativo en VHDL.

los cuales se vieron adaptados en la respuesta de ganancia de acuerdo a la descripción establecida en VHDL, uno de los aspectos más importantes corresponde a la longitud del paso que está dado por $m$ bits (con $m$ configurable a la aplicación), en esta descripción las operaciones de producto para el incremento y división para el decremento fueron tratados a partir de arreglos lógicos.

Para ello se seleccionó la concatenación en el bit menos significativo para incrementar el escalón y en el más significativo, para el correspondiente decremento, de esta manera se simplifican las operaciones aritméticas del modelo algorítmico y se mantiene la naturaleza del hardware presentado en el diagrama de bloques. Para este primer ensayo se consideró el ajuste de la ganancia de un solo elemento reflectante, pero se ha considerado un ajuste combinado.

\section{Control Óptimo de velocidad aplicado en máquinas eléctricas rotativas}

Con el objetivo de obtener los parámetros del controlador para el menor consumo de energía, garantizando los criterios de eficiencia del sistema reconfigurable, se considera el control de velocidad, aplicable a turbinas de conversión de energía, accionadas por vapor (convertidor termo-solar) o viento (convertidor eólico), motores, etc., se plantea un control óptimo, partiendo de datos de entrenamiento [2], como el ejemplo presentado en la Figura 7.

\section{Algoritmo de Entrenamiento}

Para el algoritmo de entrenamiento general MPL presentado en la Figura 8, se selecciona una propuesta de entrenamiento fraccionado, para redes de arquitectura fractal, es decir, conocidos los target de etapas intermedias del arreglo neuronal, se realiza el entrenamiento modular, para la operación matemática, concatenando las redes MPL pre-entrenadas. 

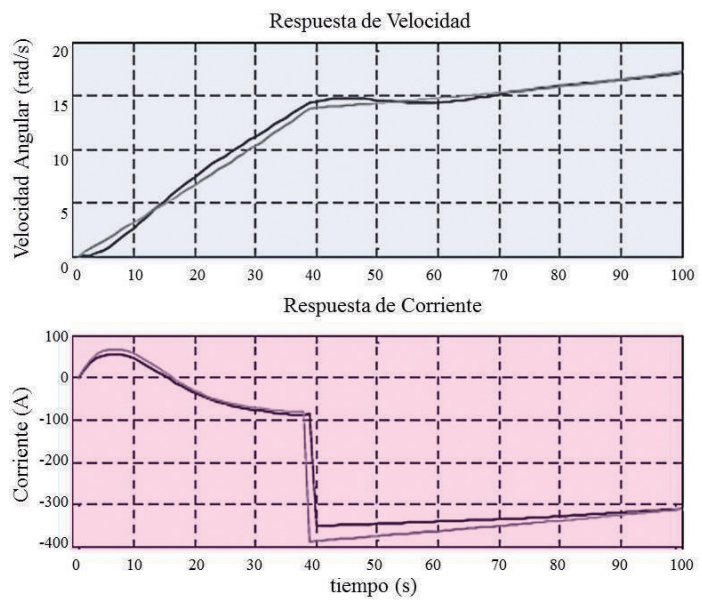

Figura 7. Data de entrenamiento Control Óptimo.

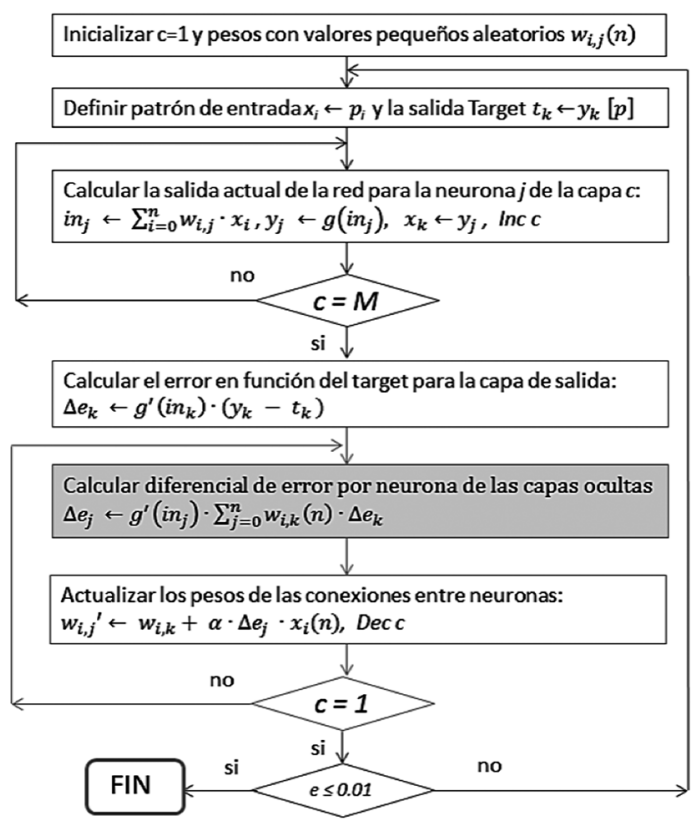

Figura 8. Algoritmo de Entrenamiento MPL.

\section{RESULTADOS}

Se desarrolló un modelo fractal, que tiene como ventaja el ajuste de los parámetros de la red en el circuito, para obtener la respuesta a la dinámica del sistema de potencia configurable, basados en criterios de eficiencia. Para el diseño del controlador neuronal se pueden aplicar dos métodos:

1) Control pre-diseño, que sea simulado a fin de obtener las respuestas como datos de entrenamiento de una red neuronal MPL estática como aproximador universal.

2) Diseño del controlador reconfigurable en circuito, a través de una sub-red neuronal modeladora del sistema a controlar concatenada con la red de control (diseño de circuito fractal).

De esta manera, se integran conceptos de inteligencia computacional para Smart Grid [30] y redes neuronales artificiales, como modelos para el diseño de los dispositivos electrónicos inteligentes sobre FPGA, que permitan optimizar la eficiencia de la red eléctrica y sistemas de potencia con generación distribuida [31], extrapolando la capacidad de reconfiguración dinámica de los dispositivos electrónicos a los sistemas de potencia.

En la Figura 9, se presenta el diseño del control reconfigurable, para módulos de energía renovable, acoplados a través de un control neuronal.

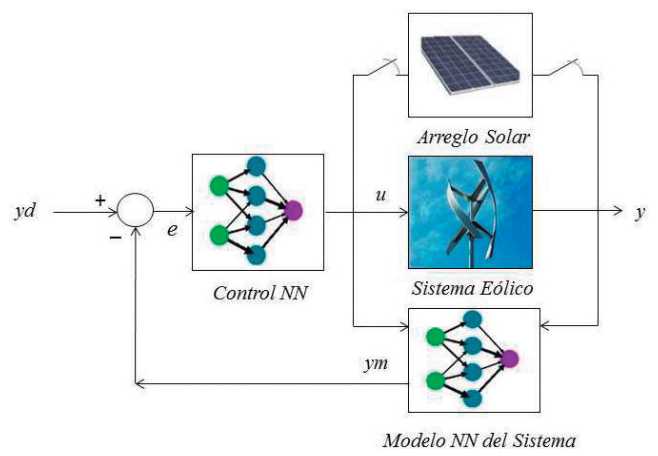

Figura 9. Esquema de Control RNN Adaptativo.

Se plantea así una red neuronal, con ganancia programable, para gestión de la potencias de cada fuente de conversión. Además de una red autoregresiva, para modelado de la turbina eólica (a partir de las curvas de velocidad/potencia).

El control neuronal fractal, permite ampliar la vida útil de los equipos, es decir solucionar la obsolescencia de los componentes del sistema de potencia, que pueden ser readaptados y actualizados, a través de reconfiguración dinámica del hardware. Adicionalmente, permite aprender el comportamiento del modelo de convertidor y optimizar su control, incluyendo conceptos de aprovechamiento del calor residual y los espacios de forma óptima (campos solares de alta concentración). Entre los objetivos se 
plantea disminuir residuos, disipación de calor, $\mathrm{CO}_{2}$ e impacto ambiental, con la aplicación de inteligencia artificial y gemelos digitales. El concepto comprende un tratamiento fractal de todos sus componentes convertidores de energía, su arquitectura, losIEDs y componentes de la matriz energética reconfigurable.

\section{Modelo Matemático de la Red Fractal}

Se obtiene el modelo matemático de la red fractal, el cual está dado por una red neuronal embebida en una red neuronal de control, como se presenta en la ecuación (7).

$$
\begin{aligned}
& u_{c}(t)=f_{R} \sum_{i=0}^{R} w_{c, i} *\left(y_{d}(t)-y_{m}(t)\right) \\
& \text { con } \\
& y_{m}(t)=f_{n} \sum_{j=0}^{R} w_{m, i} \cdot u_{c}(t)+w_{m, i} \cdot y(t)
\end{aligned}
$$

Al sustituir $y_{m}(t)$ por la salida de la subred neuronal que modela el sistema, es decir la salida predictiva, se puede expresar de la forma de la ecuación (8).

$$
u_{c}(t)=f_{R} \sum_{i=0}^{R} w_{c, i} *\left[y_{d}-f_{n} \sum_{j=0}^{R} w_{m, i}\left(u_{c}+y\right)\right]
$$

Donde, $u_{c}(t)$ es la entrada al sistema controlada, $w_{c, i}$ corresponde a la matriz de pesos sinápticos de la red neuronal del controlador, $w_{m, i}$ es la matriz de pesos sinápticos de la red neuronal modeladora del sistema.

Esta propuesta plantea un pre-diseño, en el entrenamiento neuronal, para finalmente configurar la RNA sobre hardware, con los parámetros calculados. Se define una red dinámica auto-regresiva, para el modelado del sistema, obteniendo la salida predictiva, que interactúa con la red del controlador, esta salida del modelo $y_{m}(t)$, operada por la matriz de pesos sinápticos de la capa de salida del controlador. La configuración de la red neuronal es presentada en la Figura 10.

Se puede observar una red dinámica multicapa, con interacción hacia adelante y con realimentación de la capa de salida hacia la capa oculta. La descripción en VHDL de los modelos generalizados de redes neuronales, con señales de dimensión configurable, como se presentan en la Tabla 2.

Esta red diseñada puede ser reconfigurada como una red MPL (multicapa) estática, para la implementación
Tabla 2. Descripción VHDL de la Red Fractal.

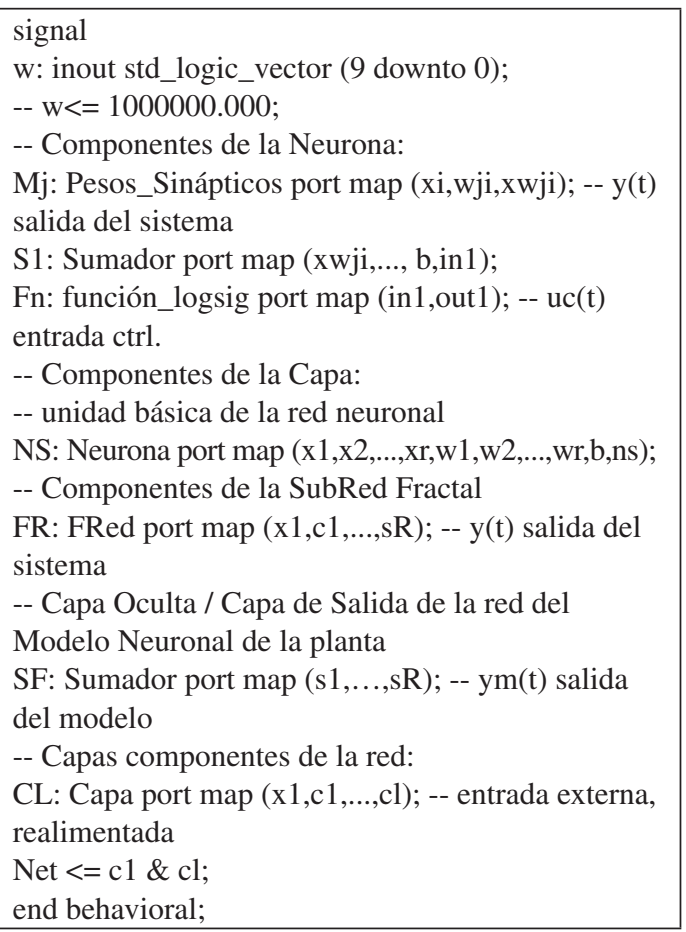

del control prediseñado, una red auto-regresiva NARX dinámica, para el modelado de los sistemas no lineales y una red fractal, que comprende una red neuronal de control con una sub-red para el modelado del proceso, esto con solo reasignar las señales a los elementos descritos en VHDL que corresponden a la base del modelo de red neuronal reconfigurable para control avanzado.

Para el entrenamiento de este modelo de control neuronal se diseñó un algoritmo de adaptativo optimizado para hardware en VHDL, el cual adapta los pesos sinápticos por medio de operaciones lógicas y comparación del error instantáneo de la red neuronal, este algoritmo es presentado en la Tabla 3.

En la condición inicial se seleccionó el bit más significativo en cada parámetro, partiendo de esta primera aproximación se obtiene un error, cuyo signo determina la próxima aproximación y se continua el proceso de iteraciones. El criterio detallado en [8] puede ser modificado por desplazamiento en registros de forma simultánea, entre parámetros por iteración con el objetivo de aumentar la velocidad de la convergencia, de los pesos sinápticos y polarización, para establecer el ajuste fino. 


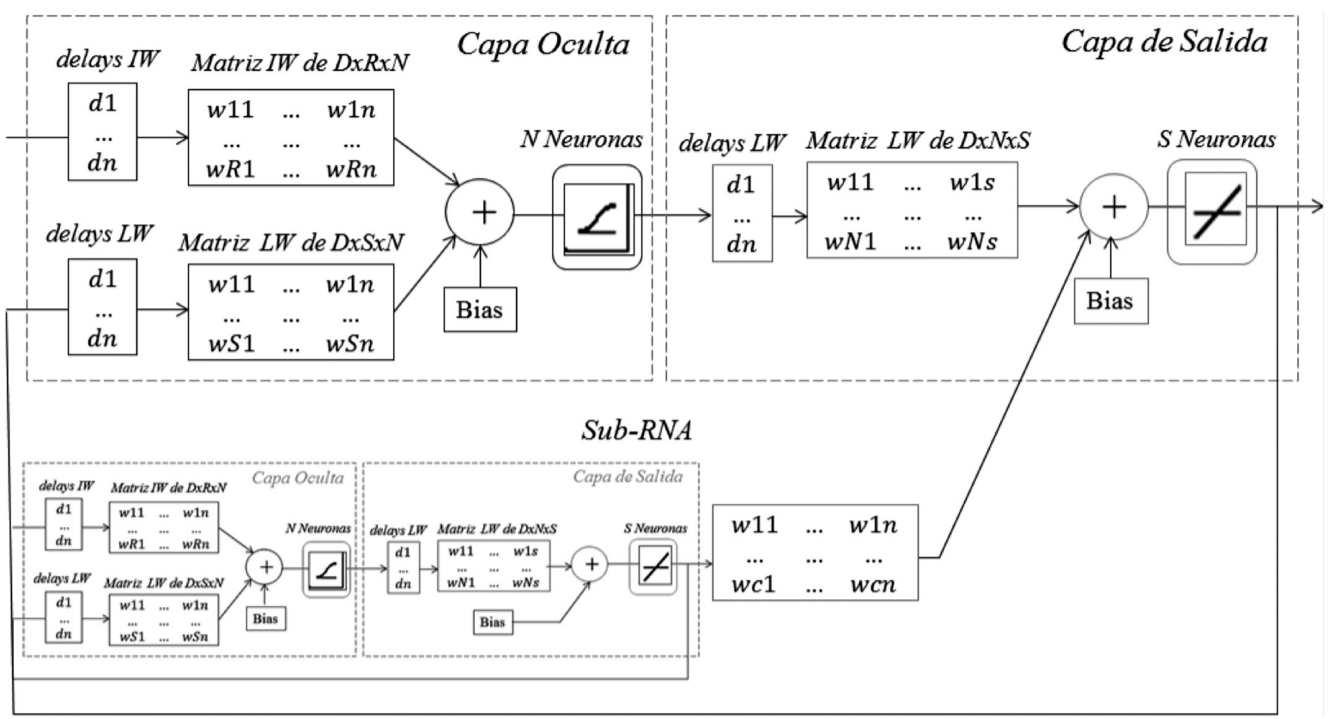

Figura 10. Red Multicapa Dinámica Auto-Regresiva

Tabla 3. Algoritmo Adaptativo en VHDL.

\begin{tabular}{|l|}
\hline \multicolumn{1}{|c|}{ Aproximación con arquitectura SAR1: } \\
\hline if clk $=$ ' 1 ' and clk'event then \\
$\mathrm{SAR}<=$ Reg $>>1$-- prueba bit más significativo \\
$\mathrm{wp}<=\mathrm{w}$ or SAR; -- cálculo del peso de prueba \\
en $<=\mathrm{T}$ - $\left(\mathrm{x}{ }^{*} \mathrm{wp}+\mathrm{b}\right)$; -- cálculo del error para $\mathrm{w}$ \\
prueba \\
if $\mathrm{e}<\mathrm{en}$ then -- $e$ error anterior y en error parcial \\
actual \\
$\mathrm{n} \_\mathrm{SAR}<=$ not (SAR); -- resetear el bit de prueba \\
$\mathrm{w}<=$ wp and n_SAR; -- actualización condicionada \\
-- actualiza e, para la próxima aproximación \\
sucesiva para w \\
\hline
\end{tabular}

Con este método de entrenamiento orientado a hardware, a través de operaciones lógicas con menor complejidad que las operaciones aritméticas convencionales se logra un modelo de control competitivo con esquemas de control neuronal.

\section{Unidades Neuro-Fractales - UNF}

Se definió una unidad de operación, en este caso un multiplicador en campos finitos, como elemento componente del arreglo neuronal. Este elemento es modelado, entrenado y evaluado para su aplicación en el sistema neuronal generalizado.

Una vez definido una operación fractal como unidad neuronal básica, se establecen los target (salidas deseadas) para el entrenamiento fraccionado. La representación de la arquitectura definida para esta red neuronal básica es presentada en la Figura 11.

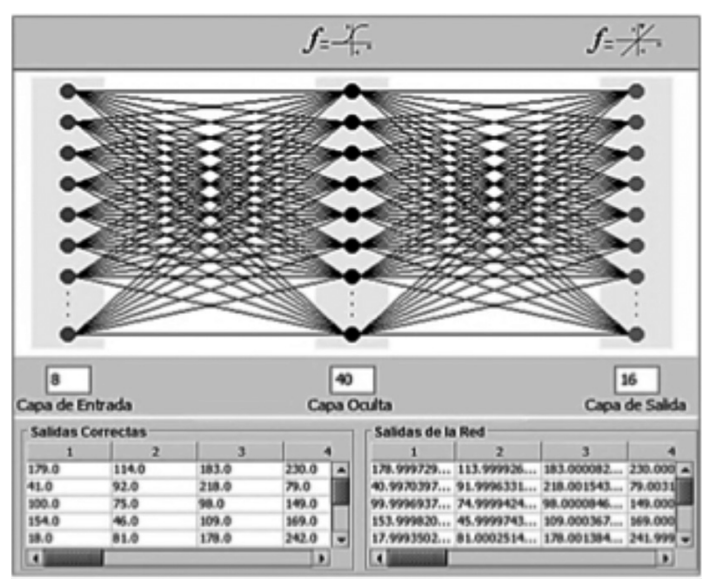

Figura 11. Red de la Unidad Neuro-Fractal.

Se puede observar que la salida corresponde con el target, la salida esperada con la que se entrenó la red neuronal, teniendo un error de $1.03 \mathrm{E}-6$, los datos de entrenamiento son presentados en la Tabla 4. El número de neuronas empleadas en el diseño de la UNF, corresponde con 40 neuronas multiplexadas por cada salida $k$, de manera que su implementación consume 40+k neuronas, una línea de retardo de 16 elementos, y un sumador. 
Tabla 4. Datos de Entrenamiento de la UNF.

\begin{tabular}{|l|c|}
\hline \multicolumn{2}{|c|}{ Entrenamiento } \\
\hline Algoritmo de Aprendizaje & Descenso del Gradiente \\
\hline Épocas del Entrenamiento & 2000 \\
\hline Constante de Aprendizaje & 0.001 \\
\hline Error de Validación & $7.24 \mathrm{E}-7$ \\
\hline Esquemas de Modelos Neuronales & Entrenamiento \\
\hline Modelo Neuronal Predictivo & en circuito \\
\hline Pre-diseñado de la Red Neuronal & en software \\
\hline Redes CNN & en circuito \\
\hline RNN Fractal* & en circuito \\
\hline
\end{tabular}

*El modelo fractal es configurable en tiempo de ejecución.

Una vez definida la unidad neuronal fractal, este modelo generalizado permite una estimación de recursos de hardware (según la métrica de elementos del dispositivos FPGA) en función de las UNF previamente definidas, que pueden ser pre-entrenadas. Se obtuvo la utilización de recursos del dispositivo de hardware $x c 4 v l x 15-12 s f 363$, a través de la herramienta ISE 11.1 presentados en la Tabla 5.

Tabla 5. Consumo de Recursos Arreglo de UNF.

\begin{tabular}{|c|c|c|c|}
\hline \multicolumn{4}{|c|}{ Multiplicador DF8 FPGA: xc4vlx15-12sf363 ISE 11.1 } \\
\hline Utilización Lógica & \begin{tabular}{l|l} 
Utilizado & D
\end{tabular} & Disponible & $\%$ \\
\hline Slices & 35 & 6144 & 0 \\
\hline LUTs 4 & 62 & 12288 & 0 \\
\hline IOBs & 24 & 240 & 10 \\
\hline Unidad Básica 8bits & 8bits & \multicolumn{2}{|c|}{ Report } \\
\hline $\begin{array}{l}\text { Registros (8bits) } \\
\text { XOR } \\
\text { LUTS }(4 x 4)\end{array}$ & $\begin{array}{l}22 \\
47 \\
\end{array}$ & \multicolumn{2}{|c|}{62} \\
\hline Esquema & 32 Neuronas & \multicolumn{2}{|c|}{ Red Aprox. } \\
\hline $\begin{array}{l}\text { Registros (8bits) } \\
\text { XOR } \\
\text { LUTs }(4 \times 4)\end{array}$ & $\begin{array}{r}32 \\
686 \\
432\end{array}$ & \multicolumn{2}{|c|}{$\begin{array}{l}* \text { capas } \\
n * \text { LUTn* }{ }^{*} \text { capas } \\
9 * \text { LUTn }{ }^{*} \text { capas }\end{array}$} \\
\hline
\end{tabular}

Los reportes de consumo de recursos de la unidad neuro-fractal básica, de la red neuronal genérica, permite calcular los arreglos neuronales parametrizables. Podemos observar un menor consumo de recursos de hardware y menor complejidad de cómputo, a la vez que soporta un entrenamiento con algoritmos adaptativos en circuito, lo que lo hace un modelo aplicable en el desarrollo y gestión de la infraestructura energética, a través de dispositivos electrónicos reconfigurables, para la inserción de energías renovables en los sistemas de potencia con nuevas tecnologías.

Finalmente, se obtuvo el modelo del arreglo neuronal fractal, en función de este elemento, que puede ser expresada en relación a un sistema de funciones iteradas, dado por la ecuación (9).

$$
y(n)=f_{n}\left(\ldots f_{2} \sum_{i=0}^{R} w_{2, i} * f_{1} \sum_{j=0}^{R}\left(w_{1, i} \cdot u(t)\right)\right)
$$

Con $\mathrm{w}_{2, \mathrm{i}}$ los pesos de la red modelo $\mathrm{y}_{\mathrm{w}} \mathrm{i}$ los pesos de la unidad neuro-fractal básica. En función del modelo matemático se puede realizar la estimación de recursos para las aplicaciones basadas en modelos fractales [32].

\section{CONCLUSIONES}

Gracias al modelo de control reconfigurable desarrollado, a través de redes neuronales fractales, se obtiene un aporte para la optimización de aplicaciones sostenibles. Se logra simplificar el modelo matemático propio del control óptimo en relación a los parámetros del sistema y criterios de eficiencia energética. Igualmente, se han considerado las técnicas de entrenamiento fraccionado como solución al modelo de control neuronal fractal, donde se plantea el aprendizaje modular para la red.

De esta manera, el modelo de control neuronal sobre hardware reconfigurable, simplifica la etapa de modelado de sistemas de potencia y diseño del esquema de control avanzado, facilitando el entrenamiento en circuito y la adaptación óptima en tiempo real. Ampliando así el campo de aplicación para configuración de arreglos de micro-convertidores, así como para mecanismos de seguimiento en centrales de energías renovables, basados en dispositivos electrónicos inteligentes.

Incorporar el concepto de neuro-fractal ofrece grandes ventajas en las que se extrapola la tecnología de hardware reconfigurable desde el nivel de la electrónica de control a los sistemas eléctricos de potencia, para el manejo adaptativo de las aplicaciones y reconfiguración dinámica de los esquemas modulares en la arquitectura del circuito de potencia. El diseño del control neuronal sobre tecnología FPGA, amplía las tendencias 
en digitalización de centrales eléctricas, a la vez que representa un valioso aporte para esta área de investigación.

Observando la importancia de señalar el nuevo concepto de Control Electrónico para el Sistema Reconfigurable - CESR, que presenta esquemas de control generalizados para diversos tipos de fuentes de energía, que pueden ser configurados a la medida, con funciones de identificación de sistemas y entrenamiento en línea de la red neuronal. Permitiendo tratar la planta como un sistema de potencia inteligente, ajustado a partir de condiciones del entorno, incorporar esquemas regenerativos de energía con realimentación controlada, de forma altamente eficiente.

Se puede destacar que el modelo matemáticológico desarrollado de control neuronal fractal, con tecnología de hardware promueve la generación de nuevos conocimientos de control avanzado para sistemas reconfigurables, de dinámica compleja. Lo que representa un aporte para la inserción de fuentes de energía renovables, redes inteligentes y eficiencia energética, ampliando el alcance de éstos, con la disminución de costos operativos, a partir de reutilización, reciclaje y readaptación. Aspecto importante para promover la migración a tecnologías sostenibles, hacerlas más accesibles y así obtener diseños con menor impacto ambiental, a partir del CESR.

\section{REFERENCIAS}

[1] V. Sotelo-Neyra and F. Paredes-Larroca. "Multirate-sampling adaptive controller for an induction generator driven by a wind turbine". Ingeniería, Investigación y Tecnología. Vol. 19 $\mathrm{N}^{\mathrm{o}}$ 1, pp. 51-62. 2018. ISSN 2594-0732. DOI:10.22201/fi.25940732e.2018.19n1.005

[2] C. Sandoval, K. Velazco y J. Díaz. "Accionamiento eléctrico de sistemas dinámicos a través de criterios de control óptimo". Rev. Ing e Investig. Vol. $28 \mathrm{~N}^{\mathrm{o}} 2$, pp. 66-71. 2008. ISNN: 0102-5609.

[3] P. Santis. "Diseño de Estrategias de Control Predictivo Multi-Objetivo Diseño de Estrategias de Control Predictivo MultiObjetivo". Tesis para optar al grado de Magister en Ciencia de la Ingeniería. Universidad de Chile. 2016.

[4] C. Silva, D. Neto, J. Oliveira, J. Melo, D. Barbalho and A. Avelino. "Definition of an Architecture to Configure Artificial Neural Networks Topologies Using Partial Reconfiguration in FPGA". IEEE Lat. Am. Trans. Vol. 13 N $\mathrm{N}^{\circ}$, pp. 2094-2100. 2015. ISSN: 1548-0992. DOI: $10.1109 /$ TLA.2015.7273763

[5] J.A. Flórez-Vargas y R. Alzate-Castaño. "Control híbrido conmutado como alternativa tecnológica para la eficiencia energética". Ing. Solidaría. Vol. 9 No 16 , pp. 81-87. 2013.

[6] C. Sandoval-Ruiz. "Adaptive Control in VHDL Applied to a Solar Oven". Rev. Colomb. Tecnol. Avanzada. Vol. $1 \mathrm{~N}^{\circ} 23$, pp. 142-147. 2014. ISSN: 1692-7257.

[7] D. Steckler, C. Nava, J. Duarte, J. Zambrano and C.E. Sandoval-Ruiz. "Design of Neural Networks on microcontrollers, applied in functional modules for an eco-park". Revista Ingeniería UC. Vol. $25 \mathrm{~N}^{\circ}$ 1, pp. 50-60. 2018. ISSN: 1316-6832.

[8] C. Sandoval-Ruiz. "Modelo Neuro-Adaptativo en VHDL, basado en Circuitos NLFSR, para Control de un Sistema Inteligente de Tecnología Sostenible". Rev. Universidad, Cienc. y Tecnol. Vol. 21 № 85, pp. 140-149. 2017. ISSN 2542-3401

[9] C. Sandoval-Ruiz. "Sistema Eco-Adaptativo integrado en elementos arquitectónicos con tecnología sostenible". Rev. Electrónica Científica Perspectiva. Vol. 4 No 8, pp. 96109. 2015.

[10] C. Sandoval-Ruiz. "Plataforma Reconfigurable de Investigación aplicada a Movilidad Sostenible". Rev. Universidad, Cienc. y Tecnol. Vol. $20 \mathrm{~N}^{\circ} 78$, pp. 35-41. 2016. ISSN 2542-3401

[11] I. Gonzalez-Garcia. "Control Neuronal de un Generador de Inducción para Generación Eólica”. Instituto Polictécnico Nacional, D.F. México. 2005.

[12] D. Villa, C. Martin, F. Villanueva, F. Moya and J. Lopez. "A dynamically reconfigurable architecture for smart grids". IEEE Trans. Consum. Electron. Vol. $57 \mathrm{~N}^{\circ}$ 2, pp. 411-419. 2011. DOI: 10.1109/TCE.2011.5955174

[13] K. Beleño, J. Berrio, A. Pardo y G. Oscar. "Diseño de una smart grid para un sistema híbrido de energía Design of a smart grid for a hybrid power system". Prospect. Vol. 11 No 2, pp. 94-101. 2013. 
[14] L. Hernández. "Aplicación de Técnicas no lineales y otros paradigmas en Smart Grid/ MicroGrid/Virtual Power Plant”. Tesis Doctoral. Universidad de Valladolid. Madrid, España. 2016.

[15] R. Real. "Integración de dispositivos electrónicos inteligentes en smart grid". Tesis Doctoral. Universidad de Cordova. 2016.

[16] T. García, J. Carlos y L. López. "Técnicas de Inteligencia Artificial aplicadas a la Red Eléctrica Inteligente - Smart Grid". Novótica. Vol. 213, pp. 29-34. 2011. ISSN: 0211-2124

[17] R. Real-Calvo, A. Moreno-Muñoz, V. Pallares-Lopez, M.J. Gonzalez- Redondo, I.M. Moreno-García y E.J. PalaciosGarcía. "Sistema Electrónico Inteligente para el Control de la Interconexión entre Equipamiento de Generación Distribuida y la Red Eléctrica". Rev. Iberoam. Automática e Informática Ind. RIAI. Vol. $14 \mathrm{~N}^{\mathrm{o}}$ 1, pp. 56-69. 2017. ISSN: 1697-7912. DOI: 10.1016/j. riai.2016.11.002

[18] C. Troncoso y A. Suárez. "Control del Nivel de Pulpa en un Circuito de Flotación utilizando una Estrategia de Control Predictivo". Rev. Iberoam. Automática e Informática Ind. RIAI. Vol. $14 \mathrm{~N}^{\circ} 1$, pp. 234-245. 2017. ISSN: 1697-7912. DOI: 10.1016/j.riai.2017.04.003

[19] M.T. Hagan, H.B. Demuth and M.H. Beale. “Neural Network Design". Vol. 2. 2014.

[20] C.E. Sandoval-Ruiz. "Análisis de Circuitos Fractales y Modelado a través de Sistema de Funciones Iteradas para VHDL". Rev. Ciencia e Ingeniería. Vol. $38 \mathrm{~N}^{\circ}$ 1, pp. 3-16. 2017. ISSN: 1316-7081.

[21] C. Sandoval-Ruiz. "Modelo de Estructuras Reconfigurables con Registro Desplazamiento, para Lenguaje Descriptor de Hardware VHDL". Rev. Fac Ing UCV. Vol. $31 \mathrm{~N}^{\circ} 3$, pp. 109-118. 2016. ISSN: 2443-4477.

[22] C.E. Sandoval-Ruiz. "VHDL Model of configurable neural networks applied to decoding in cognitive radio". Rev. Ingeniería UC. Vol. $24 \mathrm{~N}^{\circ} 3$, pp. 290-301. 2017. ISSN: 1316-6832.

[23] J. Castellanos, C. Sandoval and M. Azpurua. "A FPGA implementation of a LMS adaptative algorithm for smart antenna arrays". Rev. Técnica la Fac. Ing. la Univ. Zulia. Vol. 37 №3, pp. 270-278. 2014. ISSN: 0254-0770.
[24] E.D. De la Rosa M. “El aprendizaje profundo para la identificación de sistemas no lineales". Centro de Investigación y de estudio Avanzados del Instituto Politécnico Nacional. México, D.F. 2014.

[25] C.E. Sandoval-Ruiz. "Códigos Reed Solomon para Sistemas Distribuidos de Energías Renovables y Smart Grids a través de Dispositivos Electrónicos Inteligentes sobre Tecnología FPGA". Memorias. Investig. en Ing. Vol. $16 \mathrm{~N}^{\circ} 1$, pp. 37-54. 2018. ISSN: 2301-1092.

[26] C. Sandoval Ruiz. "Plataforma de Gestión, Investigación y Formación en Tecnologías Sostenibles, para soporte de un Laboratorio Remoto". Rev. Eduweb, Univ. Carabobo. Vol. 10 No 1, pp. 79-92. 2016. ISSN: 1856-7576.

[27] W. Fernández Ravanales and A. Rodriguez Garcia. "Analysis of the Regulatory Requirements for the Smart Grid in Chile" IEEE Lat. Am. Trans. Vol. $15 \mathrm{~N}^{\circ}$ 1, pp. 13-20. 2017. ISSN: 1548-0992.

[28] W. Fernández y A. Rodríguez. “OFDM óptimo para la comunicación bidireccional en las redes eléctricas inteligentes". Ingeniare Revista Chilena de Ingeniería. Vol. $26 \mathrm{~N}^{\circ} 1$, pp. 43-53. 2018. ISSN 0718-3305. DOI: 10.4067/S0718-33052018000100043.

[29] H. Kaschel Cárcamo y E. Viera Riquelme. "Desarrollo de protocolo de comunicaciones para redes HAN en SmartGrids Development". Ingeniare Revista Chilena de Ingeniería. Vol. $25 \mathrm{~N}^{\mathrm{o}}$ 1, pp. 15-27. 2017. ISSN 0718-3305. DOI: $10.4067 /$ S0718-33052017000100015.

[30] R.G. Harley and J. Liang. "Computational Intelligence in Smart Grids". IEEE Symposium Series on Computational Intelligence (SSCI). Vol. 1, pp. 1-8. 2011.

[31] C. Sandoval-Ruiz. "Control de Micro-Redes de Energía Renovable a través de estructuras LFCS Reconfigurables en VHDL". Revista Ciencia y Tecnología. Vol. 18, pp. 71-86. 2018. ISSN: 1850-0870. DOI: 10.18682/ cyt.v1i18.847.

[32] C. Sandoval-Ruiz. "Métodos Numéricos en Diferencias Finitas para la Estimación de Recursos de Hardware FPGA en arquitecturas LFSR $(n, k)$ Fractales". Ingeniería Investigación y Tecnología. Vol. XX $\mathrm{N}^{\circ} 03$, pp. 1-10. 2019. ISSN: 2594-0732. DOI: 10.22201/fi.25940732e.2019.20n3.032. 